\title{
Au cœur de l'Europe, le Quichotte
}

\section{Marie-Hélène Maux}

\section{(2) OpenEdition}

Journals

Édition électronique

URL : http://journals.openedition.org/rbnu/665

DOI : 10.4000/rbnu.665

ISSN : 2679-6104

\section{Éditeur}

Bibliothèque nationale et universitaire de Strasbourg

\section{Édition imprimée}

Date de publication : 1 novembre 2017

Pagination : 36-39

ISBN : 9782859230678

ISSN : 2109-2761

\section{Référence électronique}

Marie-Hélène Maux, «Au cœur de l'Europe, le Quichotte », La Revue de la BNU [En ligne], 16 | 2017, mis en ligne le 01 juillet 2019, consulté le 23 décembre 2020. URL : http://journals.openedition.org/rbnu/ 665 ; DOI : https://doi.org/10.4000/rbnu.665

\section{(c) $10(0$}

La Revue de la BNU est mise à disposition selon les termes de la Licence Creative Commons Attribution - Pas d'Utilisation Commerciale - Partage dans les Mêmes Conditions 4.0 International. 


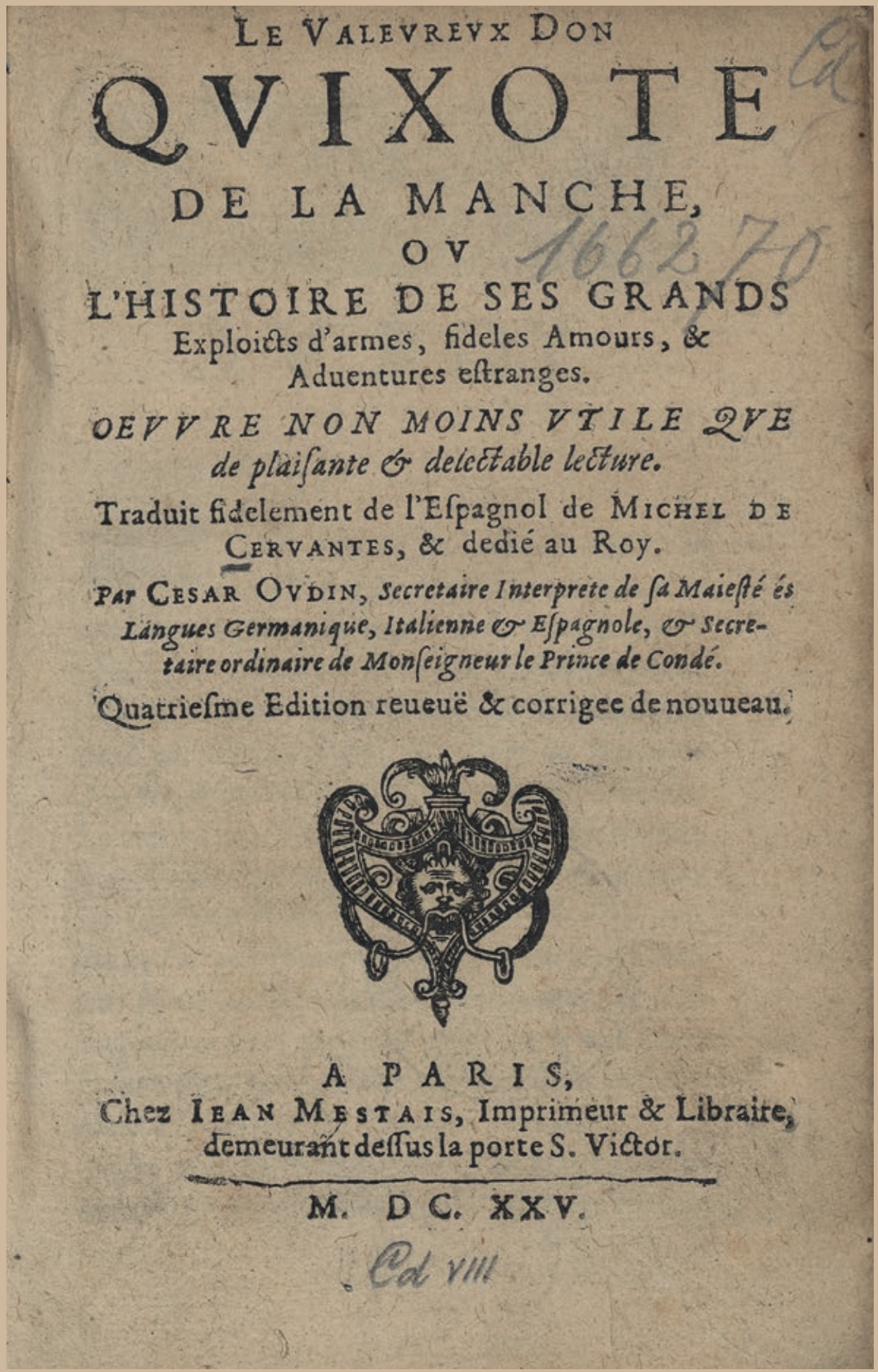




\section{ESPAGNE \\ AU CEEUR DE L'EUROPE, \\ LE QUICHOTTE \\ PAR MARIE-HÉLÈNE MAUX}

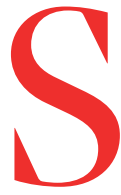

oixante ans après la signature du traité de Rome et malgré les difficultés que traverse actuellement l’Union européenne, les citoyens des différents pays ont appris à se connaître, à vivre ensemble et à partager un nombre croissant de valeurs et de références culturelles communes. Cependant, les stéréotypes, les lieux communs et les images d'Epinal n'ont pas disparu pour autant. Il suffit de prononcer le nom d'un pays pour générer automatiquement dans l'imaginaire collectif une série d'images à propos des modes de vie, des mentalités, de la culture. Pour l'Espagne, le symbole qui s'est imposé est un personnage littéraire, issu de l'imagination de Miguel de Cervantes : « el ingenioso hidalgo don Quijote de la Mancha », et la langue espagnole est désormais désignée en français comme « la langue de Cervantès ».

Don Quichotte " naît » en 1605, date de la publication du premier tome. Le second volume paraît dix ans plus tard. Les aventures du nobliau de province à l'esprit perturbé par la lecture des romans de chevalerie médiévaux, au point qu'il décide de ressusciter la chevalerie errante et de partir sur les routes, sont devenues universelles; et pourtant don Quichotte semble incarner la quintessence de l'Espagne, ou même, de façon encore plus réductrice, de la Castille. Les Espagnols se sont immédiatement appropriés le Quichotte, comme le prouvent les extensions de privilège et les multiples rééditions dès la première année de sa publication ${ }^{1}$.

Le succès de l'ouvrage trouve rapidement un écho au-delà des frontières, et les traducteurs se mettent très tôt à l'œuvre. En 1612, Thomas Shelton fait paraître la première version anglaise, The delightful Historie of the most Ingenious Knight Don Quixote de la $M a n c h a^{2}$, puis en 1614 c'est la traduction française de César Oudin qui voit le jour sous le titre L'ingénieux don Quichotte de la Manche ; enfin, en 1622, Lorenzo Franciosini publie L'ingegnoso cittadino don Chisciotte della Mancia.

Le succès est presqu'aussi grand en France que ce qu'il avait été en Espagne. Du vivant d'Oudin, on compte quatre éditions du premier tome, 1614, 1616, 1620 et 1625. La BNU possède la dernière, publiée par Jean Mestais à Paris. C'est un volume in-octavo relié 
en parchemin. Le texte compte 736 pages numérotées, précédées par 2 pages de dédicace au roi, 10 pages de prologue et une page d'avis au lecteur ; 6 pages de table des matières non numérotées sont placées à la fin de l'ouvrage.

Diana Esteba Ramos ${ }^{3}$ décrit l'ouvrage de la façon suivante dans le catalogue du fonds hispanique ancien qu'elle a établi :

\section{LE VALEVREVX DON / QVIXOTE / DE LA MANCHE, / OV / L'HISTOIRE DE SES GRANDS / Exploicts d'armes, fideles Amours, \& / Aduentures estranges. / OEVVRE NON MOINS VTILE QVE / de plaisante \& delectable lecture. / Traduit fidelement de l'Espagnol de MICHEL DE / CERVANTES, \& dedié au Roy. / Par CESAR OVDIN, Secretaire Intreprete de sa Maiesté és / Langues Germanique, Italienne \& Espagnole, $\&$ Secre- / taire ordinaire de Monseigneur le Prince de Condé. / Quatriesme Edition reueuë \& corrigee de nouueau. / <Gravure > / A PARIS, / Chez IEAN MESTAIS, Imprimeur \& Libraire, /demeurant dessus la porte $S$. Victor. / <Filet> / M. DC. XXV.}

On note tout d'abord la place centrale qu'occupe le nom du traducteur, dont les titres sont déclinés : César Oudin avait la charge de secrétaire interprète auprès de Louis XIII (il l'avait obtenue sous Henri IV), et il était secrétaire du prince de Condé. Son activité de traducteur est plus modeste, et la première partie $\mathrm{du}$ Quichotte sera sa seule œuvre conséquente en ce domaine. Avant tout, c'est un maître de langue, auteur d'un célèbre manuel d'espagnol à l'usage des Français et de diverses publications didactiques qui mettent en regard le français et l'espagnol, à une époque où les circonstances politiques amènent la cour de France à s'intéresser à la langue d'outre-Pyrénées. C'est sûrement dans cette logique que s'inscrit sa traduction du Quichotte. Oudin était par essence un passeur, un intermédiaire entre différentes cultures, dans le cas présent entre l'Espagne et la France. C'est grâce à des personnalités comme la sienne que les frontières s'abolissent et que des valeurs communes s'instaurent. En revanche, le nom de l'auteur semble presque marginal ; il est francisé sous la forme « Michel de Cervantes », est placé à cheval sur deux lignes et sur le même plan que l'indication que l'ouvrage est "dedié au Roy ».

Oudin propose une traduction qui s'affirme comme "fidèle "; la précision apparaît dès la première édition. C'est un élément fondamental de son travail et cette revendication est pour lui une véritable obsession. Par le terme "fidèle ", on entend la volonté de ne pas trahir : il faut respecter à la fois le texte source et le public destinataire. Les Français doivent accéder au même contenu que les Espagnols. La conséquence pour le texte cible sera double : d'une part, le français est parfois lourd et même opaque, "d'une littéralité fatigante », pour citer Maurice Bardon ${ }^{4}$, et d'autre part le traducteur se verra contraint d'ajouter des notes marginales (plus de 400 dans la première édition), qui visent soit à apporter des précisions linguistiques, soit à instruire les Français de particularités péninsulaires ${ }^{5}$.

La page de titre de la quatrième édition, reproduite ici, indique que des modifications ont été apportées : «Quatriesme Edition reueüe \& corrigee de nouueau », idée reprise par le bref avis au lecteur qui annonce : « voicy la quatriesme Edition de Don Quixote de la Manche que je te presente, reveüe, corrigee, \& expurgee d'une infinité de fautes qui s'estoient escoulees par mesgarde en la premiere, seconde, \& troisiesme ». Mais c'est surtout par rapport à la première édition que les différences sont visibles. Dès la deuxième édition (Paris, Jean Foüet, 1616), le titre est modifié. Tout d'abord, l'adjectif « ingenioso », polysémique en espagnol, qui fait référence à la fois à l'intelligence et à la capacité d'invention, était initialement rendu par le calque "ingénieux ». Dès la deuxième édition, il est remplacé par "valeureux ». La perte de sens est grande, mais en adéquation avec le sous-titre suivi d'un commentaire qui explicite désormais le titre principal : L'HISTOIRE DE SES GRANDS / Exploicts d'armes, fideles Amours, \& / Aduentures estranges. / OEVVRE NON MOINS VTILE QVE / de plaisante \& delectable lecture. Il s'agit d'attirer le lecteur français par une brève synthèse du contenu et de l'assurer qu'il s'instruira en s'amusant, idéal de toute publication littéraire à l'époque classique, s'il lit les aventures d'un personnage dont l'adjectif « valeureux » laisse entendre qu'il est un héros. 
C'est donc grâce à César Oudin qu'à peine neuf ans après la publication de ses aventures dans la péninsule ibérique, les Français ont pu faire leur le personnage aux multiples facettes qui allait devenir le symbole d'une culture et plus tard d'une nation, et cet exemplaire de la quatrième édition en est un témoin précieux. Nul doute qu'il a contribué au cours des siècles à faire connaître don Quichotte et son inséparable compagnon de route Sancho Panza. Cet in-octavo, de la taille d'un livre de poche, imprimé à Paris en 1625, arrive à la Kaiserliche Universitäts- und Landesbibliothek zu Strassburg le 15 juillet 1890, en provenance de la Königliche Bibliothek de Dresde. Il faisait partie d'un lot disparate ${ }^{6}$ qui avait été acheté par la bibliothèque alsacienne. Nous ne savons rien des propriétaires précédents, seule une étiquette à moitié effacée indique qu'il a été répertorié dans la bibliothèque d'un couvent de franciscains. Comme les hommes, les livres voyagent et cet exemplaire du Quichotte, parti de Paris au $17^{\mathrm{e}}$ siècle, se trouvait deux siècles plus tard dans l'est de l'Allemagne avant que les vicissitudes de l'Histoire ne le ramènent à sa patrie d'origine. Les lecteurs successifs qui l'ont eu entre les mains ont contribué, à leur échelle, à la transformation $\mathrm{du}$ personnage cervantin en mythe et en symbole.

\section{NOTES}

1- Canavaggio, Jean, Don Quichotte, du livre au mythe, Paris, Fayard, 2005, p. 47

2- Il est cependant probable qu'une version manuscrite ait circulé en Angleterre dès les années 1607-1608.

3- Esteba Ramos, Diana, Grammaire, lexicographie et littérature espagnole : catalogue du fonds ancien de la Bibliothèque nationale et universitaire de Strasbourg (XVI et XVII e siècles), Strasbourg, Recherches, $\mathrm{n}^{\circ}$ hors-série, 2012

4- Bardon, Maurice, « Don Quichotte » en France au XVII et au XVIII siècle. 1605-1815, Paris, Librairie ancienne Honoré Champion, 1931, p. 28

5- Maux-Piovano, Marie-Hélène, « Las notas marginales en la traducción francesa del Quijote por César Oudin ", in Rivista internazionale di tecnica della traduzione, Trieste, Università, 17, 2015, p. 47-57

6- Je remercie Christophe Didier, conservateur des bibliothèques, pour ces informations. 